\title{
Preface: Colorectal Cancer Biology, Diagnosis, and Therapy
}

Colorectal cancer (CRC) is a heterogenous disease, which is the third leading cause of cancer-associated mortalities worldwide and a major health challenge globally. The primary risk factors linked with the occurrence of CRC include lifestyle choices, diet, and medical/family history of cancer. CRC is asymptomatic during the initial stages and often diagnosed at advanced metastatic stages. The CRC development is a multistep process, which is an outcome of various genetic and epigenetic alterations. Identifying diagnostic predictive and prognostic biomarkers may lead to early diagnosis of CRC and aid in diminishing its occurrence. Currently, there is an ongoing quest to develop potent and sensitive biomarkers around the world. Improved screening techniques and advanced therapeutic strategies assist in decreasing CRC incidences and mortality. However, the burden of managing this lethal malignancy is extremely severe, mainly due to its ability to develop multi-drug resistance in the tumor cell. Therefore, novel strategies that aim at targeting the tumor onset, prevention, and progression are crucial. This issue covers the morphological, diagnostic, and novel therapeutic approaches for the management of CRC. Furthermore, it also focused on current research data pertaining to the therapeutic and medicinal strategies that are available for the therapy of CRC.

Herein, the issue starts to focus on understanding the progression of CRC at the biological and molecular levels, particularly genetic and epigenetic alterations. The origin of a mutation determines the category of CRC: familial, sporadic, or hereditary. This forms the basis for the individualized therapeutic strategies for CRC patients and targets the dysregulated pathways, such as PI3K/ Akt, KRAS, Wnt, and MAPK. The tumor microenvironment of CRC is always attributed to identifing novel genetic and epigenetic biomarkers that aid in the early diagnosis of CRC progression. Moreover, the gut microbiota have been considered as commensal residents in the gut and contribute to CRC pre-carcinogenesis. The pathogenesis of CRC is supported by few bacterial species, including Clostridium septicum, Helicobacter pylori, and Escherichia coli. Thus, understanding and comparing the composition of gut microbiota of a CRC patient and a healthy individual may assist in developing novel therapeutic strategies against the microbiota manipulation. Moreover, many studies have determined that a profound change in the bacterial strain or the microbiota persists in the intestinal tract, which is due to the consumption of unhealthy diets comprised of red meat and considered a common risk factor for developing CRC. The interaction between nutrients consumed and the microbiota greatly impacts the intestines, causing disorders such as chronic inflammatory bowel disease and CRC. Additionally, researchers are now encouraging the use of plant-based diets, including fibers, micronutrients, and bioactive compounds that are rich in antioxidants and possess anti-cancerous properties. Thus, consuming certain functional foods would decrease the occurrence of CRC as well regulate its progression.

In this issue, the authors focus on discussing diet, active components, and the role of food in treating CRC. Similarly, phytochemicals are naturally derived components and potential inhibitors of CRC progression. The conventional adjuvant therapies, such as radiotherapy and chemotherapy, are efficient but limited due to their toxic effects and chemoresistance developed by the tumor cells. Phytochemicals can sensitize the tumor cells to chemo drugs. For instance, 5-FU in combination with phytochemicals (such as curcumin or resveratrol) sensitizes CRC cells and enhances the efficacy of the drug. Furthermore, reports compiled current information related to the advanced therapeutic strategies, such as immunotherapy, targeted therapy, and combinational therapies, that enhance the drug efficacy. Targeted therapy focuses on the pathways involved in angiogenesis, EGFR and VEGFR, to control metastasis and widely used to develop personalized medicine. 
This issue explores the major factors that influence the disparities in CRC. Finally, this issue centered current research on factors like age, family, history, and response to therapy while also screening differences that are demonstrated among various racial/ethical groups, including African Americans and non-Hispanic Whites of the United States. This epidemiological data could form the basis for developing individualized therapies according to the patient's condition. Thus, the presented therapeutic strategies reflect contemporary and modern research concepts. I am honored to present this issue to the scientific community with the hope that it will benefit cancer patients.

I acknowledge my gratitude to all of the contributors, language professionals, technical staff, and the Editor-in-Chief, Professor Benjamin Bonavida.

\section{Guest Editor:}

Dr. Ganji Purnachandra Nagaraju, PhD, DSc, FAACC

Department of Hematology and Medical Oncology

Emory University School of Medicine, Atlanta, GA 


\section{About the Guest Editor}

Dr. Ganji Purnachandra Nagaraju is a faculty member in the Department of Hematology and Medical Oncology at Emory University School of Medicine. He obtained his MSc and $\mathrm{PhD}$, both in biotechnology, from Sri Venkateswara University in Tirupati, Andhra Pradesh, India, and his DSc from Berhampur University in Berhampur, Odisha, India. Dr. Nagaraju's research focuses on translational research in gastrointestinal malignancies. He has published more than 95 research and review articles in highly respected international journals and presented more than 50 abstracts at various national and international conferences. He is an author and editor of several books. Dr. Nagaraju is an editorial board member of several internationally recognized academic journals and an associate member of the Discovery and Developmental Therapeutics research program at the Winship Cancer Institute. He is also member of the Association of Scientists of Indian Origin in America, the Society for Integrative and

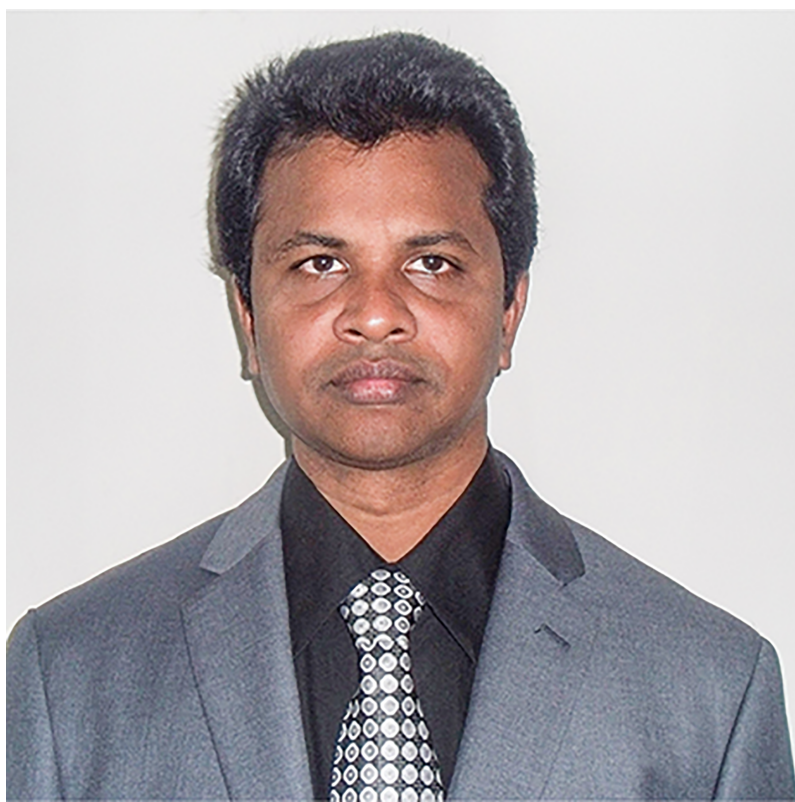

Comparative Biology, the Science Advisory Board, The RNA Society, and the American Association of Cancer Research. 
